\title{
Microscopic effective reaction theory for direct nuclear reactions
}

\author{
Kazuyuki Ogata ${ }^{1, a}$, Kosho Minomo ${ }^{1}$, Masakazu Toyokawa², Michio Kohno ${ }^{1}$, Takuma Matsumoto ${ }^{2}$, \\ Masanobu Yahiro ${ }^{2}$, Yuma Kikuchi ${ }^{1,3}$, Tokuro Fukui ${ }^{1, b}$, Kazuki Yoshida ${ }^{1}$, and Kazuhito \\ Mizuyama $^{1, c}$ \\ ${ }^{1}$ Research Center for Nuclear Physics, Osaka University, Ibaraki, Osaka 567-0047, Japan \\ ${ }^{2}$ Department of Physics, Kyushu University, Fukuoka 812-8581, Japan \\ ${ }^{3}$ RIKEN Nishina Center, RIKEN, Wako 351-0198, Japan
}

\begin{abstract}
Some recent activities with the microscopic effective reaction theory (MERT) on elastic, inelastic, breakup, transfer, and knockout processes are reviewed briefly. As a possible alternative to MERT, a description of elastic and inelastic scattering with the continuum particle-vibration coupling (cPVC) method is also discussed.
\end{abstract}

\section{Introduction}

Nowadays, a microscopic description of various types of direct nuclear reactions based on the multiple scattering theory (MST) for nucleus-nucleus scattering [1] is feasible. A key prescription of this approach is, as adopted in the traditional direct reaction studies [2], to select essential degrees of freedom in describing a direct reaction process of interest with a desired accuracy. For example, if a breakup reaction of a projectile $\mathrm{P}$ into two fragments $c$ and $v$ with a target nucleus $\mathrm{A}$ is considered, it will be natural to adopt an $\mathrm{A}+c+v$ three-body model. The model Hamiltonian contains A-c and A- $v$ one-body potentials. For them, we use the nucleus-nucleus MST. Then the three-body scattering problem can accurately be solved by sophisticated direct reaction models such as the continuumdiscretized coupled-channels method (CDCC) [5-7], the dynamical eikonal approach (DEA) [8, 9], and Faddeev-Alt-Grassberger-Sandhas (Faddeev-AGS) theory [10, 11]. This will be one of the most efficient microscopic approaches to direct nuclear reactions of various kind. We call it the microscopic effective reaction theory $(M E R T)$. Extension of MERT to four-body breakup reactions [12-14] and a reaction process involving an excitation of a constituent of $\mathrm{P}[15,16]$ will be straightforward.

Obviously, the concept of MERT is different from that of the ab-initio calculation of nuclear reactions [3, 4], i.e., MERT is a theoretical framework in a model space. This means, at least at this moment, MERT is not designed to describe all the phenomena triggered by an incident particle colliding with a target nucleus. However, once a model space is appropriately set, MERT can be regarded as a reaction theory having a predictive power for scattering observables of various projectiles and target nuclei in a wide range of incident energies.

\footnotetext{
ae-mail: kazuyuki@rcnp.osaka-u.ac.jp

bPresent address: Nuclear Data Center, Japan Atomic Energy Agency, Tokai, Ibaraki 319-1195, Japan

${ }^{\mathrm{c}}$ Present address: Nuclear Data Center, Japan Atomic Energy Agency, Tokai, Ibaraki 319-1195, Japan
} 
In Sect. 2, we briefly review some recent activities with MERT. The application of the continuum particle-vibration coupling (cPVC) method to elastic and inelastic scattering processes, as a possible alternative to MERT, is also discussed. Section 3 is devoted to a summary and future perspective.

\section{Direct reaction studies with MERT}

\subsection{Three nucleon force effect on elastic and inelastic scattering}

One of the hot topics in nuclear physics is the three nucleon force (3NF) effect on nuclear reaction observables. We adopt the chiral effective field theory (Ch-EFT) $[17,18]$ to treat the nucleon-nucleon $(N N)$ two-body and three-body interactions on the same footing. We then construct an $N N g$-matrix interaction in the infinite nuclear matter taking into account the $3 \mathrm{NF}$ effect $[19,20]$. As discussed in Ref. [7], the $g$-matrix interaction is assumed to be a good approximation to the $N N$ effective interaction appearing in the nucleus-nucleus MST [1], except at very low energies.

We show in Fig. 1 the cross sections and analyzing powers in proton elastic scattering on several target nuclei at $65 \mathrm{MeV}$ [21]. It should be noted that the calculation contains no free adjustable parameters. The results in Fig. 1 therefore show the predictability of the $g$-matrix folding calculation to proton elastic scattering for not only the cross section but also the spin observable. We do not find a significant $3 \mathrm{NF}$ effect on these observables because the proton elastic scattering probes mainly the surface region of the target nucleus in which the density is low and the $3 \mathrm{NF}$ is expected to be small. On the other hand, we see a somewhat large $3 \mathrm{NF}$ effect on nucleus-nucleus elastic and inelastic scattering cross sections [24, 25]. For details, see the references above as well as the proceedings paper for CNR* 15 by K. Minomo and his collaborators.
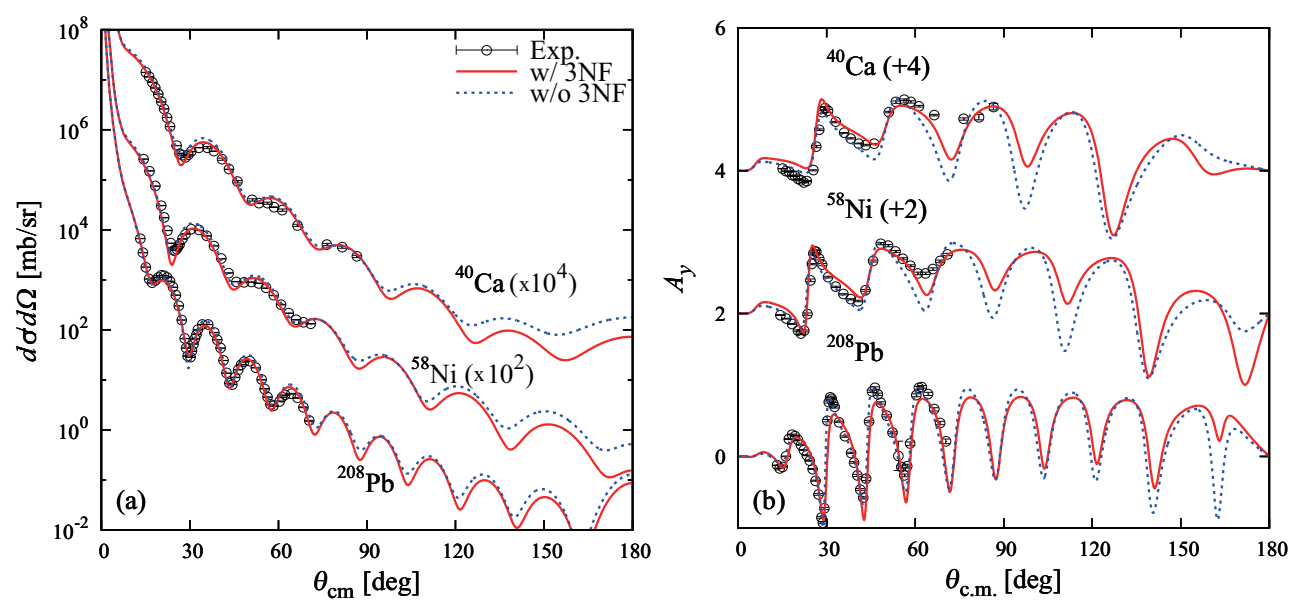

Figure 1. (a) Cross sections and (b) analyzing powers for proton elastic scattering at $65 \mathrm{MeV}$ compared with the experimental data [22, 23]. The solid (dashed) lines represent the results with (without) the $3 \mathrm{NF}$ effect. Each cross section is multiplied by the number beside the line, whereas each analyzing power is shifted up by the number. This figure is taken from Ref. [21]. 


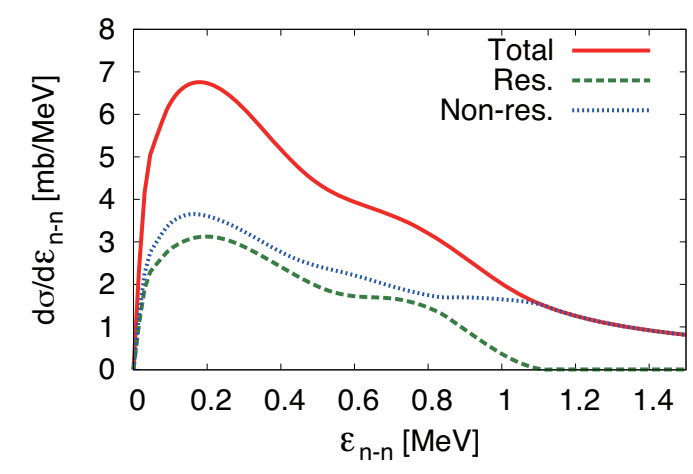

Figure 2. $n-n$ invariant mass spectra [26] (solid line). The decay cross sections for the $2_{1}^{+}$resonant state and the nonresonant three-body continuum states are shown by the dashed and dotted lines, respectively.

\subsection{Decay mode of the $2_{1}^{+}$sate of ${ }^{6} \mathrm{He}$}

Another important subject in the physics of unstable nuclei is the decay mode of a three-body system that is not stable against the particle emission. In Ref. [26], we studied the $\alpha+n+n$ three-body decay of the $2_{1}^{+}$sate of ${ }^{6} \mathrm{He}$ populated by the ${ }^{6} \mathrm{He}$ breakup process on the ${ }^{12} \mathrm{C}$ nucleus at $250 \mathrm{MeV} /$ nucleon. The essential feature of this study is both the formation and decay of the particle-unbound state of ${ }^{6} \mathrm{He}$ are described on the same footing. For this purpose we have developed a new framework that is an extended version of the method of complexed-scaled solutions of the Lippmann-Schwinger equation (CSLS) [27] utilizing the transition matrix for the four-body breakup evaluated by CDCC [12, 13]. The $n-{ }^{12} \mathrm{C}$ and $\alpha-{ }^{12} \mathrm{C}$ distorting potentials are evaluated by the $g$-matrix folding model. The CDCCCSLS calculation can thus be regarded as an application of MERT to a four-body breakup/decay process.

The solid line in Fig. 2 shows the $n$ - $n$ invariant mass spectra. One sees a peak at around $0.2 \mathrm{MeV}$ of the $n-n$ relative energy $\left(\epsilon_{n n}\right)$ and a shoulder structure at $\epsilon_{n n} \sim 0.8 \mathrm{MeV}$. The former is due to the $n-n$ final state interaction (FSI) in the decaying process, which has already been found in the Coulomb breakup of ${ }^{6} \mathrm{He}$ [28]. On the other hand, the latter appears only in the nuclear breakup of ${ }^{6} \mathrm{He}$, in which the $2_{1}^{+}$resonant state is expected to be well populated. We then put a gate on the three-body breakup energy $\epsilon$ corresponding to the $2_{1}^{+}$state, i.e., $\epsilon=0.98 \pm 0.14 \mathrm{MeV}$. The result is shown by the dashed line that is regarded as the decay cross section from the $2_{1}^{+}$resonance. The dotted line is obtained by subtracting the dashed line from the solid line, which corresponds to the decay of the nonresonant continuum states. One sees that the low-energy peak appears in both lines, whereas the shoulder structure is found only in the dashed line. It should be noted that since $\epsilon$ is restricted to around $1 \mathrm{MeV}$ in the resonant decay (dashed line), at the shoulder the three-body energy is almost exhausted by the $n-n$ relative energy.

One can therefore conclude that there are two decay modes of the $2_{1}^{+}$state of ${ }^{6} \mathrm{He}$. One is the decay mode in which the two neutrons are emitted in correlation with each other. The other is the mode in which the two neutrons are emitted to the opposite directions. The latter, the back-to-back decay mode, will be important in particular, because it is free from the FSI and may suggest a di-neutron structure in the $2_{1}^{+}$state of ${ }^{6} \mathrm{He}$. For more detailed discussion, the reader is referred to Ref. [26].

\subsection{Three-body dynamics in the ${ }^{8} \mathbf{B}(d, n)^{9} \mathbf{C}$ reaction}

Direct nuclear reactions are sometimes utilized as an alternative way of extracting a cross section that is difficult to measure directly. In Ref. [29] the cross section of the ${ }^{8} \mathrm{~B}(d, n)^{9} \mathrm{C}$ reaction was measured and thereby that of the ${ }^{8} \mathrm{~B}(p, \gamma){ }^{9} \mathrm{C}$ process was extracted; the so-called asymptotic normalization 


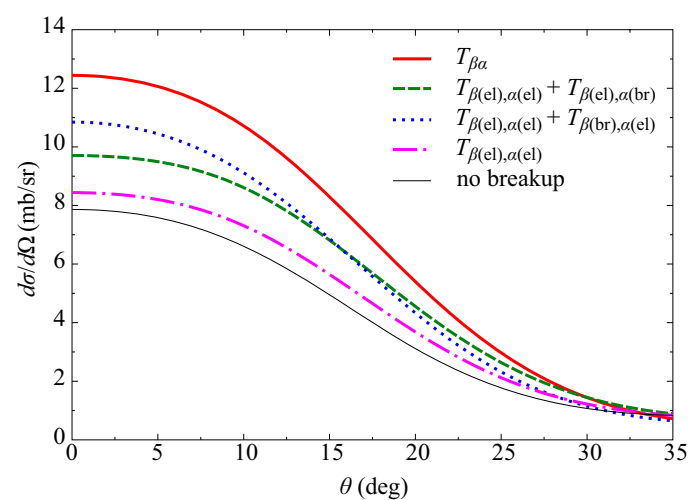

Figure 3. Cross section of the ${ }^{8} \mathrm{~B}(d, n)^{9} \mathrm{C}$ reaction at 14.4 MeV/nucleon [31]. The meaning of each line is given in the text.

coefficient (ANC) method [30] was adopted to relate the two cross sections. However, the reaction model used to analyze the ${ }^{8} \mathrm{~B}(d, n){ }^{9} \mathrm{C}$ reaction was DWBA, in which the breakup channels of $d$ and ${ }^{9} \mathrm{C}$ were not included explicitly.

Recently, we have re-analyzed the ${ }^{8} \mathrm{~B}(d, n)^{9} \mathrm{C}$ reaction at $14.4 \mathrm{MeV} /$ nucleon by means of the CDCC-BA framework [31]. The key feature is that the breakup channels of both $d$ and ${ }^{9} \mathrm{C}$ are taken into account by CDCC. Figure 3 shows the resulting cross sections as a function of the center-of-mass scattering angle. The thick solid line is the result of CDCC-BA, the dashed (dotted) line corresponds to the calculation with including the breakup channels of $d\left({ }^{9} \mathrm{C}\right)$ only, and the dash-dotted line shows the result with neglecting all the breakup channels. Note that in these four calculations breakup channels are taken into account by CDCC in evaluating the three-body wave functions in the initial and final channels; the selection of channels are done in the evaluation of the transition matrix for the transfer process. On the other hand, the thin solid line is the result obtained with neglecting the breakup channels in the calculation of the three-body wave functions.

The small difference between the thin solid line and the dash-dotted line suggests that the threebody wave function in the elastic channel is not affected by the coupling to the breakup states, in each of the initial and final channels. Therefore the difference between the thick solid line and the dashdotted line is due to the contribution of the transfer process through the breakup states of $d$ or ${ }^{9} \mathrm{C}$. It is found that the inclusion of the breakup states of $d\left({ }^{9} \mathrm{C}\right)$ increases the transfer cross section by about $23 \%(38 \%)$. This finding will strongly suggest the importance of the breakup states of both $d$ and ${ }^{9} \mathrm{C}$ in the analysis of the ${ }^{8} \mathrm{~B}(d, n)^{9} \mathrm{C}$ reaction at $14.4 \mathrm{MeV} /$ nucleon, to reliably determine the cross section of the ${ }^{8} \mathrm{~B}(p, \gamma){ }^{9} \mathrm{C}$ process. Consequently, however, the ${ }^{8} \mathrm{~B}(p, \gamma){ }^{9} \mathrm{C}$ cross section decreased by about $51 \%$ and the result is significantly different from the value extracted by ${ }^{9} \mathrm{C}$ breakup reactions [32]. Further investigation will be necessary to draw a conclusion on the ${ }^{8} \mathrm{~B}(p, \gamma){ }^{9} \mathrm{C}$ cross section.

Another notice is that in Ref. [31] a phenomenological nucleon-nucleus optical potential was adopted. This is because that the reliability of the $g$-matrix folding model will be questionable at around $10 \mathrm{MeV}$. A microscopic framework that provides a reliable optical potential at lower energies will be very important; the cPVC method [33] (see Sect. 2.5) and the dispersive optical model (DOM) [34, 35] will be utilized for this purpose.

\subsection{Asymmetry of parallel momentum distribution of $(p, p N)$ reaction residues}

Elucidation of the single particle (s.p.) properties of nuclei and the underlying many-body correlations is one of the most important goals of nuclear physics. Nucleon knockout reactions have widely been used to study the s.p. nature of nuclei. In Refs. [36, 37], a systematics of the so-called reduction 
factor $R_{S}$ for knockout reactions was discussed; $R_{S}$ is defined by the ratio of the spectroscopic factor $S$ experimentally determined to that obtained by a shell model (SM) calculation. If $R_{S}$ is unity, it implies that the SM perfectly describes the s.p. property of the nucleus. If $R_{S}$ is lower than unity, a missing correlation in the SM calculation is suggested. Through $\left(e, e^{\prime} p\right)$ studies, $R_{S}$ was found to be around $0.6-0.7$ for stable nuclei. This is mainly because that the short-range correlation is not taken into account in the standard SM calculations. However, it was shown in Refs. [36, 37] that $R_{S}$ is around $0.2-0.4$ for the proton (neutron) s.p. state in neutron (proton) rich nuclei. This suggests a surprisingly large amount of missing correlations in such cases. Their finding can be summarized as a strong $\Delta S$ dependence of $R_{S}$, where $\Delta S$ is the difference between the proton and neutron separation energies.

On the other hand, systematic studies of $R_{S}$ with nucleon transfer reactions [38, 39] found that its $\Delta S$ dependence is much weaker than in Refs. [36, 37]. Furthermore, it was shown in Ref. [40] that if the intranuclear cascade (INC) model is adopted, $R_{S}$ extracted from one neutron knockout process from ${ }^{14} \mathrm{O}$ completely deviates from the systematics shown in Refs. [36, 37]. These counterarguments suggest that $R_{S}$ depends on reaction processes and/or reaction models adopted.

It should be noted that in the analysis of nucleon knockout reactions discussed in Refs. [36, 37], the Glauber model [41, 42] was adopted. The Glauber model is based on the eikonal and adiabatic (sudden) approximations, and the latter is valid only when the energy and momentum transfer $(\omega-q)$ is small. Since $R_{S} \sim 0.2-0.4$ is found when a tightly-bound nucleon is knocked out, the reliability of the Glauber model is not very clear. In fact, in such cases the parallel momentum distribution (PMD) of the reaction residue shows an asymmetric shape, whereas the result of the Glauber model is always symmetric because of the adiabatic approximation [40]. The asymmetry of the PMD can thus be a clue about the reliability of the Glauber model, and that to the understanding of the reaction (-model) dependence of $R_{S}$. However, the mechanism that develops the asymmetry of the PMD has not been understood well.

In Ref. [43], we have clarified how the asymmetric PMD is developed in the ${ }^{14} \mathrm{O}(p, p n){ }^{13} \mathrm{O}$ at $100 \mathrm{MeV} /$ nucleon. The distorted wave impulse approximation (DWIA) with the eikonal approximation to the distorted waves is adopted as a reaction model; the DWIA does not rely on the adiabatic approximation and safely applied to the knockout process for the tightly-bound neutron in ${ }^{14} \mathrm{O}$ having the separation energy of $23.2 \mathrm{MeV}$.

First, we show the role of the phase-volume (PV) that guarantees the energy and momentum conservation. For clear discussion, we work in the plane-wave limit, i.e., the PWIA is adopted. The solid line in the left panel of Fig. 4 shows the PWIA result without the PV, which can be regarded as
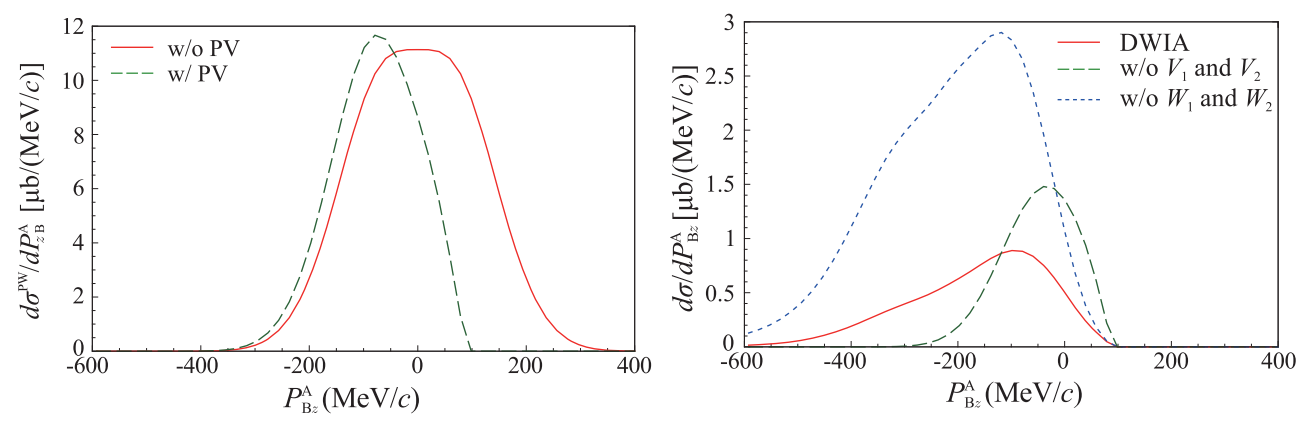

Figure 4. $\mathrm{PMD}$ of ${ }^{14} \mathrm{O}(p, p n){ }^{13} \mathrm{O}$ at $100 \mathrm{MeV} /$ nucleon [43]. See the text for detail. 
a Glauber-like calculation. As expected, the PMD is purely symmetric. The inclusion of the PV gives a quite sharp cut of the PMD on the high momentum side, which results in a narrowing of the width of the PMD, and a reduction of the integrated cross section.

We then discuss the distortion effect. There are two types of distortion: one is the absorption caused by the imaginary part $(W)$ of the optical potential $(U)$ and the other is the attraction by the real part $(V)$ of $U$. As one sees from the two dashed lines in the left and right panels, the absorption reduces the absolute value of the PMD but hardly changes its shape. On top of that, the attraction develops the low momentum tail of the PMD (the solid line in the right panel). This can be understood as follows. In the projectile-rest frame, the "target" proton comes from the $+z$ direction and hit the neutron in ${ }^{14} \mathrm{O}$. After the collision, the proton and the struck neutron are emitted to the $-z$ direction, and the reaction residue $\left({ }^{13} \mathrm{O}\right)$ feels an attraction from the two nucleons. Thus, ${ }^{13} \mathrm{O}$ prefers to have a momentum to the $-z$ direction, which results in the well-developed low momentum tail of the PMD. It should be remarked that the attraction effect does not change the integrated value of the PMD but significantly reduces its peak height. In other words, if a reaction model without the attraction effect is adopted, the peak height of the PMD will be overestimated. Consequently, a smaller value of the "experimental" $S$, hence $R_{S}$, will be extracted.

Thus, the PV and attraction effects are found to generate the asymmetry of the PMD knockout reactions with a proton target; in fact, the asymmetric PMD reflects the asymmetry in the three-body kinematics. These two effects, which cannot be taken into account in the Glauber model calculation, are expected to exist also in knockout reactions with nucleus targets. Inclusion of them in the analysis of these reactions will be interesting and important. Alternatively, systematic studies of $R_{S}$ using the $(p, p N)$ reactions will be highly desirable; DWIA based on MERT will play an important role for this purpose. For more detailed discussion, see Ref. [43].

\section{5 cPVC description of scattering observables}

As an alternative to MERT, one may apply a nuclear many-body model to scattering problems. In Ref. [33], the continuum particle-vibration coupling (cPVC) method was developed; the cPVC method treats the collective mode and the s.p. motion of individual nucleons simultaneously. We have applied the cPVC method to the neutron scattering on ${ }^{16} \mathrm{O}$ below $30 \mathrm{MeV}$ [44]. A distinguished feature of the calculation is that the $N N$ effective interaction adopted $\left(\mathrm{SkM}^{*}\right)$ has no imaginary part. In other words, the absorption in the $n^{-16} \mathrm{O}$ scattering is described by a coupling to the channels that are explicitly taken into account. This means there is no flux "loss" out of the model space adopted in the cPVC calculation.

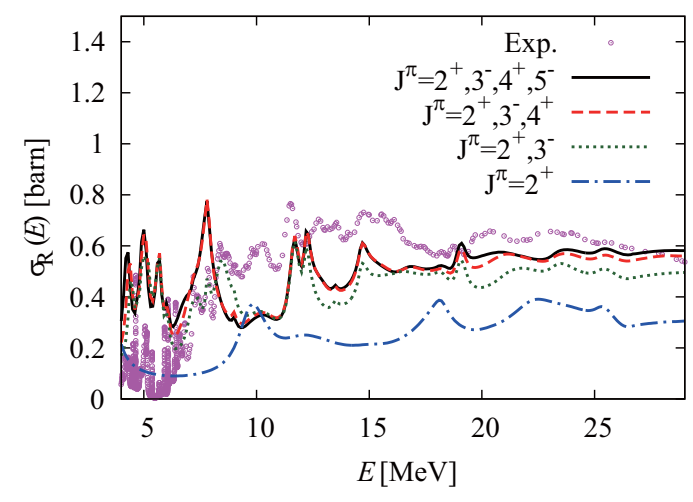

Figure 5. Total reaction cross section of neutron on ${ }^{16} \mathrm{O}$ [44]. The solid, dashed, dotted, and dash-dotted lines correspond to the calculation with the maximum multipolarity of 5, 4, 3, and 2 of the vibration mode, respectively. Experimental data are taken from Ref. [45]. 
Figure 5 shows the total reaction cross section $\sigma_{\mathrm{R}}$. It should be remarked that the peak structure of $\sigma_{\mathrm{R}}$ is reproduced quite well, which suggests the cPVC calculation can describe the doorway states of the $n+{ }^{16} \mathrm{O}$ system. It is obvious that the $g$-matrix folding model cannot reproduce the energy dependence of $\sigma_{\mathrm{R}}$ in this energy region. The cPVC method has been successfully applied to also the proton inelastic scattering on ${ }^{24} \mathrm{O}$ at $62 \mathrm{MeV} /$ nucleon [46].

\section{Summary and future perspective}

We have briefly reviewed some direct nuclear reaction studies by means of the microscopic effective reaction theory (MERT). The application of the continuum particle-vibration coupling (cPVC) method to low-energy scattering was also introduced. The microscopic description of various reaction processes is an interesting and challenging subject of theoretical nuclear physics. It is also important for an application to nuclear technology. Recently, a nuclear transmutation study for reduction and resource recycle of high level radioactive wastes has launched as an impulsing paradigm change through disruptive technologies (ImPACT) program. Development of MERT for multi-nucleon knockout and transfer reactions will be indispensable in understanding the measured cross section data of long-lived fission products (LLFPs) in inverse kinematics, and to generate neutron reaction data of LLFPs theoretically. In future, as the next step toward the description of compound nuclear reactions, it will also be important to develop MERT for pre-equilibrium processes; the semiclassical distorted wave model (SCDW) [47-51] will be suitable for this purpose.

\section{Acknowledgment}

This work has been supported in part by Grant-in-Aid of the Japan Society for the Promotion of Science (JSPS) and by the ImPACT Program of the Council for Science, Technology and Innovation (Cabinet Office, Government of Japan).

\section{References}

[1] M. Yahiro, K. Minomo, K. Ogata, and M. Kawai, Prog. Theor. Phys. 120, 767 (2008).

[2] G. R. Satchler, Direct Nuclear Reactions (Oxfrod University Press, 1983).

[3] P. Navrátil, S. Quaglioni, I. Stetcu, and B. R. Barrett, J. Phys. G 36, 083101 (2009).

[4] P. Navrátil and S. Quaglioni, Phys. Rev. Lett. 108, 042503 (2012).

[5] M. Kamimura, M. Yahiro, Y. Iseri, Y. Sakuragi, H. Kameyama, and M. Kawai, Prog. Theor. Phys. Suppl. 89, 1 (1986).

[6] N. Austern, Y. Iseri, M. Kamimura, M. Kawai, G. Rawitscher, and M. Yahiro, Phys. Rep. 154, 125 (1987).

[7] M. Yahiro, K. Ogata, T. Matsumoto, and K. Minomo, Prog. Theor. Exp. Phys. 2012, 01A206 (2012).

[8] D. Baye, P. Capel, and G. Goldstein, Phys. Rev. Lett. 95, 082502 (2005).

[9] G. Goldstein, D. Baye, and P. Capel, Phys. Rev. C 73, 024602 (2006).

[10] L. D. Faddeev, Zh. Eksp. Theor. Fiz. 39, 1459 (1960) [Sov. Phys. JETP 12, 1014 (1961)].

[11] E. O. Alt, P. Grassberger, and W. Sandhas, Nucl. Phys. B 2, 167 (1967).

[12] T. Matsumoto, E. Hiyama, K. Ogata, Y. Iseri, M. Kamimura, S. Chiba, and M. Yahiro, Phys. Rev. C 70, 061601(R) (2004). 
[13] T. Matsumoto, T. Egami, K. Ogata, Y. Iseri, M. Kamimura, and M. Yahiro, Phys. Rev. C 73, 051602(R) (2006).

[14] M. Rodríguez-Gallardo, J. M. Arias, J. Gómez-Camacho, R. C. Johnson, A. M. Moro, I. J. Thompson, and J. A. Tostevin, Phys. Rev. C 77, 064609 (2008).

[15] A. M. Moro and J. A. Lay, Phys. Rev. Lett. 109, 232502 (2012).

[16] J. A. Lay, A. M. Moro, J. M. Arias, and Y. Kanada-En'yo, Phys. Rev. C 89, 014333 (2014).

[17] E. Epelbaum, W. Glöckle, and Ulf-G. Meißner, Nucl. Phys. A 747, 362 (2005).

[18] E. Epelbaum, H.-W. Hammer, and Ulf-G. Meißner, Rev. Mod. Phys. 81, 1773 (2009).

[19] M. Kohno, Phys. Rev. C 88, 064005 (2013).

[20] M. Kohno, Prog. Theor. Exp. Phys. 2015, 123D02 (2015).

[21] M. Toyokawa, M. Yahiro, T. Matsumoto, K. Minomo, K. Ogata, and M. Kohno, Phys. Rev. C 92, 024618 (2015).

[22] H. Sakaguchi et al., Phys. Lett. B 89, 40 (1979).

[23] H. Sakaguchi et al., Phys. Lett. B 99, 92 (1981).

[24] K. Minomo, M. Toyokawa, M. Kohno, and M. Yahiro, Phys. Rev. C 90, 051601(R) (2014).

[25] K. Minomo, M. Kohno, and K. Ogata, Phys. Rev. C, in press [arXiv1509.04459].

[26] Y. Kikuchi, T. Matsumoto, K. Minomo, and K. Ogata, Phys. Rev. C 88, 021602(R) (2013).

[27] Y. Kikuchi, T. Myo, M. Takashina, K. Katō, and K. Ikeda: Prog. Theor. Phys. 122, 499 (2009).

[28] Y. Kikuchi, K. Katō, T. Myo, M. Takashina, and K. Ikeda: Phys. Rev. C 81, 044308 (2010).

[29] D. Beaumel et al., Phys. Lett. B 514, 226 (2001).

[30] A. M. Mukhamedzhanov and N. K. Timofeyuk, Yad. Fiz. 51, 679 (1990) [Sov. J. Nucl. Phys. 51, 431 (1990)].

[31] T. Fukui, K. Ogata, and M. Yahiro, Phys. Rev. C 91, 014604 (2014).

[32] T. Fukui, K. Ogata, K. Minomo, and M. Yahiro, Phys. Rev. C 86, 022801(R) (2012).

[33] K. Mizuyama, G. Colò, and E. Vigezzi, Phys. Rev. C 86, 034318 (2012).

[34] J. M. Mueller et al., Phys. Rev. C 83, 064605 (2011).

[35] M. H. Mahzoon, R. J. Charity, W. H. Dickhoff, H. Dussan, and S. J. Waldecker, Phys. Rev. Lett. 112, 162503 (2014).

[36] A. Gade et al., Phys. Rev. C 77, 044306 (2008).

[37] J. A. Tostevin and A. Gade, Phys. Rev. C 90, 057602 (2014).

[38] J. Lee et al., Phys. Rev. C 83, 057602 (2014).

[39] F. Flavigny et al., Phys. Rev. Lett. 110, 122503 (2013).

[40] F. Flavigny et al., Phys. Rev. Lett. 108, 252501 (2012).

[41] R. J. Glauber, in Lectures in Theoretical Physics (Interscience, New York, 1959), Vol. 1, p.315.

[42] K. Hencken, G. Bertsch, and H. Esbensen, Phys. Rev. C 54, 3043 (1996).

[43] K. Ogata, K. Yoshida, and K. Minomo, Phys. Rev. C 92, 034615 (2015).

[44] K. Mizuyama and K. Ogata, Phys. Rev. C 86, 041603(R) (2012).

[45] G. M. Hale et al., ENDF/B-VI, 10, 825 (2001).

[46] K. Mizuyama and K. Ogata, Phys. Rev. C 89, 034620 (2014).

[47] Y. L. Luo and M. Kawai, Phys. Rev. C 43, 2367 (1991).

[48] Y. Watanabe, R. Kuwata, Sun Weili, M. Higashi, H. Shinohara, M. Kohno, K. Ogata, and M. Kawai, Phys. Rev. C 59, 2136 (1999).

[49] Sun Weili, Y. Watanabe, M. Kohno, K. Ogata, and M. Kawai, Phys. Rev. C 60, 064605 (1999).

[50] K. Ogata, Y. Watanabe, Sun Weili, M. kohno, and M. Kawai, Nucl. Phys. A 703, 152 (2002). 
[51] K. Ogata, Y. Watanabe, Sun Weili, M. Kohno, and M. Kawai, Proc. of the Kyudai-RCNP international mini symposium on nuclear many-body and medium effects in nuclear interations and reactions (MEDIUM02) (Kyushu University Press, 2003) p. 231. 